
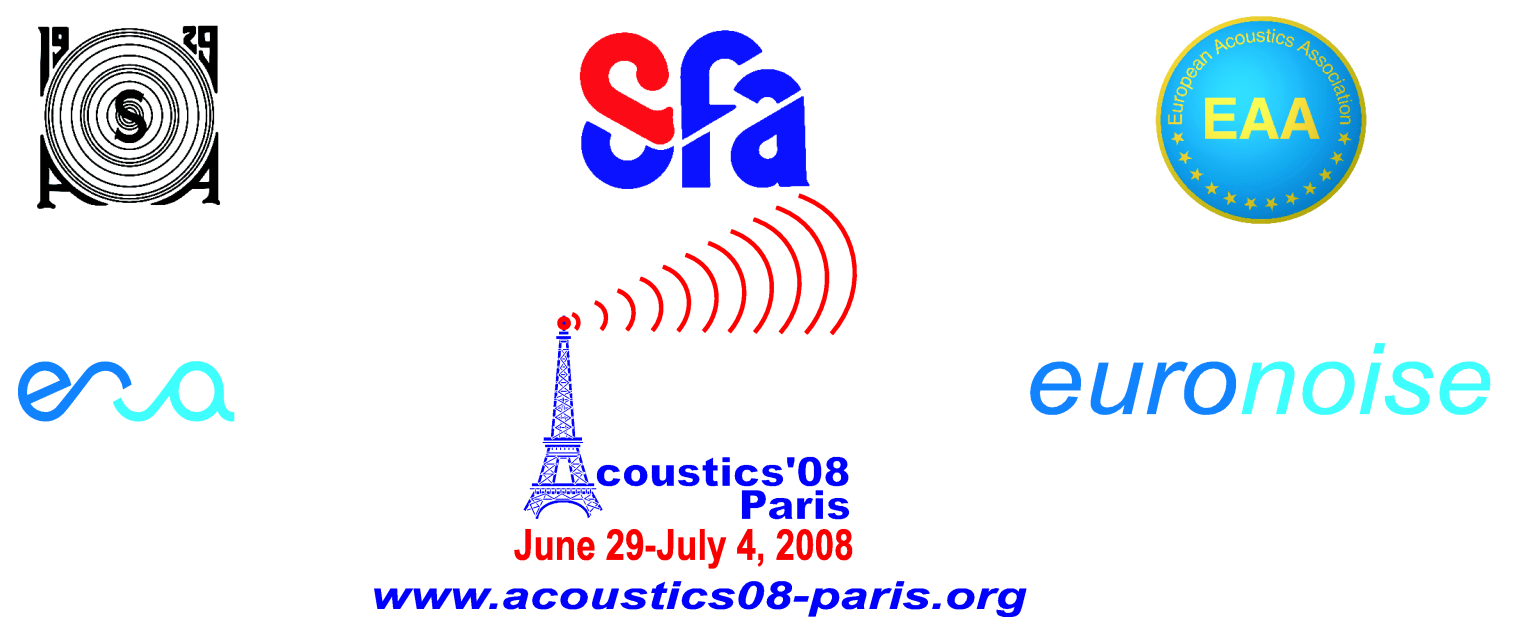

\title{
High frequency scattering measurements for mussel bed characterisation
}

\author{
M. Snellen ${ }^{\mathrm{a}}$, D. G Simons ${ }^{\mathrm{b}}$ and R. Riethmueller ${ }^{\mathrm{c}}$
}

${ }^{a}$ Delft Institute of Earth Observation and Space Systems, Delft University of Technology, Kluyverweg 1, 2629 HS Delft, Netherlands

${ }^{\mathrm{b}}$ Delft University of Technology, P.O. Box Postbus 5048, 2600 GA Delft, Netherlands

${ }^{\mathrm{c}}$ Institute for Coastal Research, GKSS Research Centre Geesthacht, Max-Planck-Str. 1, D-21502 Geesthacht, Germany

m.snellen@tudelft.nl 
exist towards seafloor classification using high-frequency backscattering measurements. The classification approach taken in this paper is a model-based classification employing backscatter data measured by a multibeam-echosounder system. The method discriminates between sediments in the most optimal way by applying the Bayes decision rule for multiple hypotheses, implicitly accounting for the backscatter strength ping-to-ping variability. The method's applicability for seafloor classification was demonstrated before by using $300 \mathrm{kHz} \mathrm{MBES}$ data collected in the Cleaver-Bank area (North Sea). The area is well-known from a geological point of view due to extensive sampling campaigns and is characterized by a wide variety of seafloor types. Here we apply the classification method to MBES data acquired in the Oosterschelde estuary (the Netherlands) which is known to contain mussel culture spots. In addition, optical recordings have been taken close to the seafloor using a video camera mounted on a towed sledge. From the video recordings estimates of mussel coverage as a function of position were derived. Analysis of the MBES results shows that they clearly reveal the presence of the mussel beds, indicating the usefulness of acoustic classification for habitat mapping. A first comparison between MBES analysis results and the video recordings is presented.

\section{Introduction}

Mapping of marine habitats is of growing importance, e.g. in the context of coastal management and for understanding the coastal ecosystems.

As coastal waters are turbid, underwater habitat surveying is done by close-by visual inspection (video, photography, divers) or by sampling, either from divers or using shipborn samplers. These techniques are partly invasive, time consuming, and provide the required information at point positions only. Another drawback is that the sampling location is not well defined and therefore not reproducible.

In literature several approaches towards the use of acoustic systems, i.e., the side scan sonar, the single beam echo sounder and the multibeam-echosounder (MBES) for remote habitat mapping using seafloor classification are described. They would allow for a much denser map of the area at limited costs. Specifically, MBES systems allow for $100 \%$ coverage of an area in limited time and are of widespread use nowadays for bathymetric mapping. Therefore, using these systems for mapping marine habitats is an attractive approach.

In addition to the travel times, the MBES provides the intensities of the backscattered signals. Correcting for losses and footprint effects allows for determining the seafloor backscatter strength for the angles at which the sound impinges on the seafloor.

In this paper we employ this backscatter strength for classifying the seafloor. Since seafloor type can vary along the MBES swath, use is made of the backscatter strength per angle. Previously the method has been applied to data acquired at the Cleaver-Bank (North Sea) ([1]). This area is characterized by a wide variety of seafloor types. The method's classification results were compared with grainsize information obtained from cores and geological maps. This comparison showed a very good agreement between the acoustic classification results, the maps and the cores, demonstrating the applicability of the method for mapping the seafloor types.

To investigate the suitability of the method for habitat mapping, here the classification approach is applied to data acquired at a mussel culture area in the Oosterschelde estuary, the Netherlands.

Section 2 provides a description of the classification approach. Section 3 gives a description of the dataset. In section 4 the results of applying the classification approach to the Oosterschelde estuary data set are presented and a comparison with the video analysis is made. The paper ends with the conclusions in section 5 .

\section{Classification approach}

\subsection{Theory}

The amplitudes of the signals as received by the MBES after scattering at the seafloor depend on the nature of the seafloor, i.e., its composition, orientation, roughness and geo-acoustic properties. In principle, therefore, these amplitudes allow for classification of the seafloor. Since shallow water MBES systems work at a few $100 \mathrm{kHz}$, the acoustic signals typically penetrate into the seafloor a few $\mathrm{cm}$ 's. Classification results using these systems therefore reflect the nature of the sediment surface.

Due to the small pulse length $T_{p}$ employed by the shallow water MBES systems (typically $\sim 100 \mu$ s) the signal footprint $A_{S}$ is also small, amounting to

$$
A_{S}=\Omega_{t x} R \frac{c T_{p}}{2 \sin \theta}
$$

with $\Omega_{t x}$ the beam aperture in the along-track direction, $\theta$ the beam angle, $c$ the average water sound speed, and $R=H / \cos \theta$, where $H$ is the water depth. For normal incidence Eq. (1) does not hold and $A_{S}=R^{2} \Omega_{t x} \Omega_{r x}$, with $\Omega_{r x}$ the beam aperture in the across-track direction.

In general the beam footprint is larger than the signal footprint, and is determined by the water depth $H$, the beam angle and the MBES transducer characteristics as follows

$$
A_{B}=\Omega_{t x} R \frac{H \Omega_{r x}}{\cos ^{2} \theta}
$$

The number of scatter pixels $N_{\mathrm{s}}$ is given by the ratio of both footprints:

$$
N_{s}(\theta)=\frac{\frac{H \Omega_{r x}}{\cos ^{2} \theta}}{\frac{c T_{p}}{2 \sin \theta}}
$$

This expression only holds for beams away from nadir. For nadir directions, i.e., $\theta \rightarrow 0^{\circ}, N_{s}=1$.

The classification method employs the backscatter values (in $\mathrm{dB}$ ) per receiver beam, i.e., backscatter values that have been obtained from averaging (or filtering) over $N_{\mathrm{s}}$ independent scatter pixels. Corrections for propagation losses and footprint are applied, and backscatter values are provided for each of the MBES beams. As explained in [1], 
the resulting backscatter values are subject to statistical fluctuations from ping to ping. If $N_{\mathrm{s}}$ is sufficiently large for the central limit theorem to hold, the backscatter values are distributed according to a normal distribution. It is assumed that the backscatter intensity of each scatter pixel in a beam is distributed according to an exponential distribution. Further, the sensitivity per beam is assumed to be uniform and seafloor type is assumed to be constant per beam footprint. Denoting the backscatter value by $y$, its mean and standard deviation then amount to

$$
\begin{gathered}
\bar{y}=10 \log _{10} \lambda-2.5 \\
\sigma_{y}=\frac{5.57}{\sqrt{N_{s}}}(\mathrm{~dB})
\end{gathered}
$$

$\lambda$ in Eq. (4) is the mean of the backscatter intensity of each individual scatter pixels.

More details on the classification approach taken for the current analysis are provided in [1].

\subsection{MBES seafloor classification}

The theory of the previous section presents the probability density function (PDF) in case backscatter measurements are taken at a single angle and at a single seafloor type. However, surveying an area in general results in measurements taken across a number of seafloor types. The number of seafloor types is often not known prior to surveying. Consequently not only the PDF (mean and standard deviation), but also the number of seafloor types present needs to be estimated. The classification method consists of the steps as described in the following.

Step 1: Nonlinear curve fitting: The algorithm starts by fitting a model to the histogram of selected measured backscatter strengths. The selected data consist of all averaged backscatter data as measured for a certain angle at the MBES system. The model that we fit to the histograms, therefore, consists of a sum of $m$ Gaussian PDFs, each PDF representing a seafloor type, i.e.,

$$
f\left(y_{j} \mid x\right)=\sum_{k=1}^{m} c_{k} \exp \left(-\frac{\left(y_{j}-\bar{y}_{k}\right)^{2}}{2 \sigma_{y_{k}}^{2}}\right)
$$

with $f\left(y_{j} \mid x\right)$ the value of the model at backscatter value $y_{j}$, and vector $x$ containing the unknown parameters, i.e., $x=\left(\bar{y}_{1}, \ldots, \bar{y}_{m}, \sigma_{y_{1}}, \ldots, \sigma_{y_{m}}, c_{1}, \ldots, c_{m}\right)^{T}$. As a measure for the match between model and measurements we consider the $\chi^{2}$ value

$$
\chi^{2}=\sum_{j=1}^{M} \frac{\left(n_{j}-f\left(y_{j} \mid x\right)\right)^{2}}{\sigma_{j}^{2}}
$$

with $M$ the number of bins in the histogram and $n_{j}$ the number of $y_{j}$ occurrences. Assuming that the $n_{j}$ are Poisson distributed, the variances $\sigma_{j}^{2}$ are equal to $n_{j}$. The unknown parameters are determined by minimizing Eq (7).

$m$, i.e., the number of detectable seafloor types present, is estimated by carrying out the fitting procedure for an increasing number of $m$, until further increasing $m$, no longer results in an improvement of the goodness of the fit. The goodness-of-fit criterion is defined as the reduced $\chi^{2}-$ value $\chi_{v}^{2}=\chi^{2} / v$ being close to one. $v=M-3 m$ is the degrees of freedom,.

Step 2: Acoustic classes identification: After step 1, both the PDF's for each of the seafloor types and the number of seafloor types are known. We assume all hypotheses equally likely. Then, by applying the Bayes decision rule for multiple $(m)$ hypotheses, a seafloor type is assigned to each measurement as follows:

$$
\text { accept } H_{k} \text { if } \underbrace{\max }_{H_{i}} f\left(y_{j} \mid H_{i}\right)=f\left(y_{j} \mid H_{k}\right)
$$

with $i=1, \ldots, m$. This means that we choose the hypothesis that, given the observation $y$, maximizes the likelihood $f(y \mid H)$. We therefore have to determine the intersections of the $m$ normal PDFs, resulting from the fitting procedure of Step 1. This results in $m$ nonoverlapping acceptance regions $A_{k}$.

Step 3: Assigning seafloor type to acoustic classes: Now, a seafloor type needs to be assigned to each of the acceptance regions. The result of this step can be accomplished by comparison of the $\bar{y}_{k}$ values with a combination of data found in the literature, model outcomes and knowledge of the surveyed area.

Step 4: Quality assessment: Based on the so-called decision matrix of the multiple-hypothesis-testing problem, probabilities of incorrect decision are determined.

Step 5: Mapping: By plotting seafloor type, e.g. with different colours representing different seafloor types, versus position, a classification map of the area is obtained.

\section{Description of the MBES data set}

The MBES survey was carried out from April 29 through May 2, 2004 as part of an extensive field campaign carried out within the framework of the EU-FP5-project "Managing Benthic Ecosystems in Relation to Physical Forcing and Environmental Constraints" (MaBenE). Details about the field campaign can be found in [2]. The surveyed area is situated in the central part of the Oosterschelde, see Fig. 1.

Depths in the surveyed area range from $\sim 4$ to $\sim 20 \mathrm{~m}$ below normal chart datum (RD/NAP, Amersfoort, Bessel). A bathymetric map obtained from the MBES measurements is shown in Fig. 2.

The MBES system used is the EM $3000^{\mathrm{TM}}$ from SimradKongsberg. It operates at a frequency of $300 \mathrm{kHz}$. The ping repetition rate amounts to $15 \mathrm{~Hz}$. The pulse length is 150 $\mu \mathrm{s}$. Both the along-track beam width and across-track beam width are $1.5^{\circ}$. In total 127 individual beams are formed over an arc of $120^{\circ}$. At $6 \mathrm{~m}$ water depth (i.e. the approximate depth of studied mussel culture plot), the footprint of each beam increases from $0.15 \mathrm{~m}$ times $0.15 \mathrm{~m}$ at nadir to $0.31 \mathrm{~m}$ along-track times $0.62 \mathrm{~m}$ across-track at the most off-nadir beams. The corresponding swath width amounts to $21 \mathrm{~m}$.

The area is known to contain a series of mussel cultures mostly situated on the banks of tidal channel. These are visible in the bathymetric map as elevated areas and clearly shaped by the activities of the mussel farmers exhibiting circular crests and troughs. The surveyed mussel sites were located on the eastern slope of the 'Brabants Vaarwater' 
channel, the water depth being around $6.2 \mathrm{~m}$ below normal chart datum. Upstream the mussel sites the seabed exhibits significant ripple structures, whereas the main channel's bed is comparably smooth.

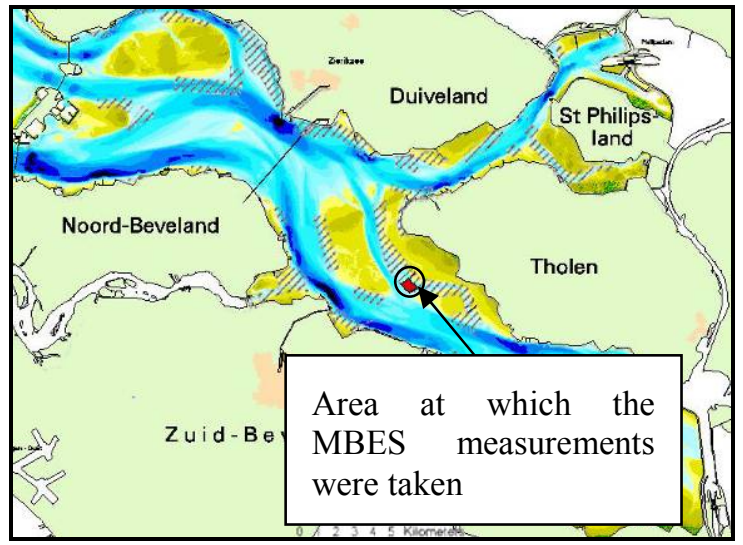

Fig. 1 The Oosterschelde area. The area indicated by a circle is the area of the experiment. The areas indicated by black lines are areas with bottom culture of mussels on cultivation spots.

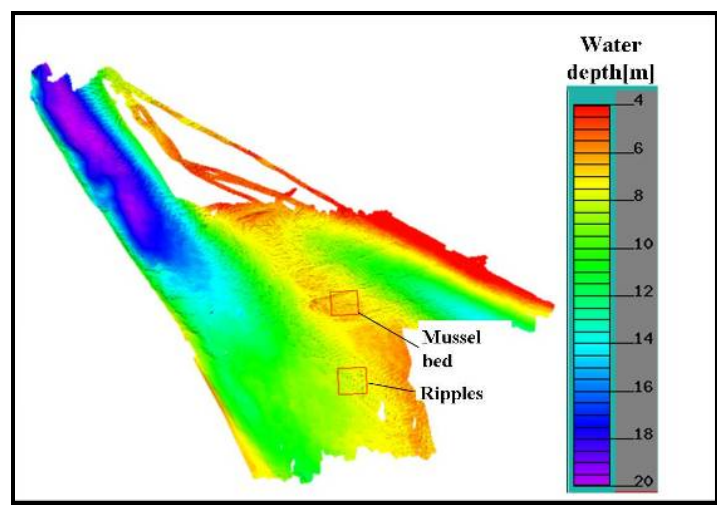

Fig. 2 Bathymetric map of the survey area. The depths are given in meters below normal chart datum (RD/NAP) [3].

In addition to the acoustic measurements, video data were acquired on selected ships tracks using an analog colour camera mounted on a sledge and towed behind the ship. During video recording, the ship travelled with a nearly constant speed of about two knots along a straight line in order to maintain the relative distance between the camera and the vessel and to minimise lateral deviations of the camera position from the central ship track. The position of the camera was known along-track by $\pm 5 \mathrm{~m}$ and acrosstrack by $\pm 2 \mathrm{~m}$. The distance of the camera to the bed was approximately $30 \mathrm{~cm}$. The visibility during the surveys was around $2 \mathrm{~m}$, a value typical for this part of the Oosterschelde ([2]), providing sufficient ambient light for unique detection of seabed features for water depths less than $10 \mathrm{~m}$. The underwater video was viewed in real time, recorded on VHS - video tapes and later converted to digital formats for further analysis. Digital video images were provided with a frequency of $25 \mathrm{~s}^{-1}$ providing images about each four centimetre along-track. The resulting images were classified due to colour and texture, employing the software package i-corder ${ }^{\circledR}$ detect from V2T-Company. Percentage mussel coverage was derived from the classified images and compared with visual inspection.

\section{Results}

\subsection{MBES classification results}

For the analysis of the MBES measurements, backscatter measurements acquired at $\theta=60^{\circ}$ are used. This angle was selected based on Eq. (3) to maximize the number of independent scatter pixels per beam footprint leading to the smallest possible values for $\sigma_{y_{k}}$ (Eq. 5).

Figure 3 shows the backscatter strengths at $\theta=60^{\circ}$ as a function of position. These values are backscatter values taken at one side of the vessel. Mapping the measurements taken at the other side of the vessel yields similar results.

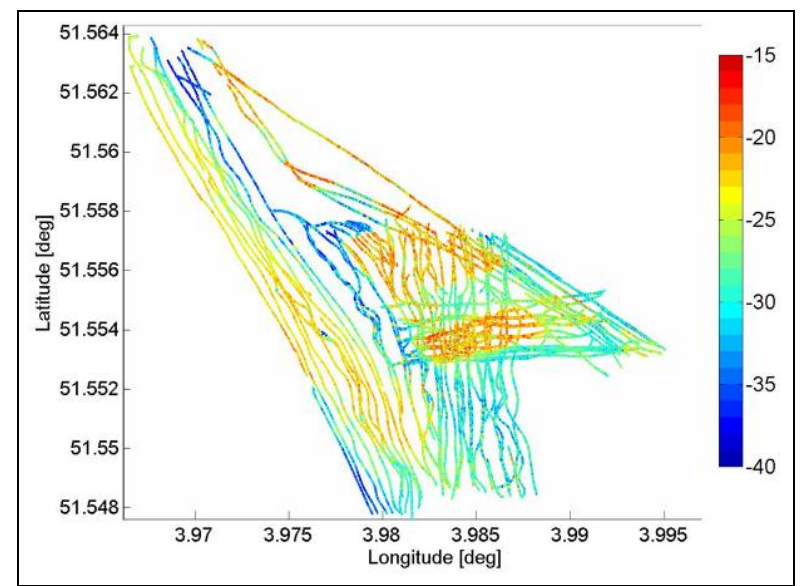

Fig. 3 Backscatter strengths in $\mathrm{dB}$ at $\theta=60^{\circ}$ along the ship track.

Figure 4 shows a histogram of the same data. As described in section 2.2, a series of Gaussian PDFs with increasing number of seafloor types $m$ is fitted to the histogram (Step 1). Starting values for the means $\bar{y}_{k}$ are evenly distributed over the range of prevailing backscatter values. The search interval for each of the means is $(-5 \mathrm{~dB},+5 \mathrm{~dB})$ around the start value. Search bounds for $\sigma_{y_{k}}$ are estimated from simulations based on Eqs. (3) and (5) by taking into account the observed distribution of water depths. Based on this information, the search bounds for the standard deviations were selected as 1 and $2.5 \mathrm{~dB}$.

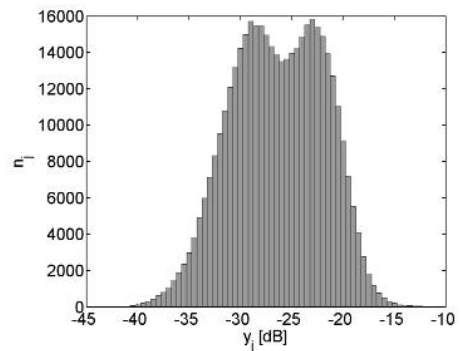

Fig.4 Histogram of backscatter strengths at a 60 degree angle.

Figure 5 shows $\chi_{v}^{2}$ as a function of $m$. The optimal value of $m$ is attained at five. Larger values of $m$ do not result in a significantly better agreement between model and measurements. This value of $m$ then can be interpreted as 
the detectable number of seafloor types contained in the data.

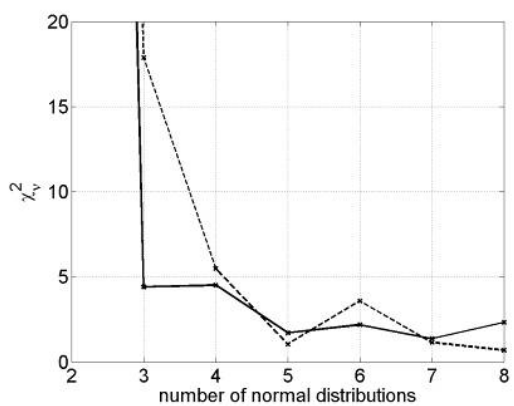

Fig.5 . Reduced $\chi^{2}$ versus $m$ for the $60^{\circ}$ backscatter data, with the solid and dashed lines corresponding to the two different sides of the MBES.

Figure 6 shows the resulting fits for $m=5$. Due to calibration uncertainties, measurements on each side of the vessel have to be treated separately. From Table 1 it is seen that similar fits are obtained for the two sides. Note that the fifth seafloor type corresponds to the highest values for the backscatter strength. Due to its relative small number of occurrences it is not visible in Fig. 6. Table 1 specifies the corresponding means and standard deviations.
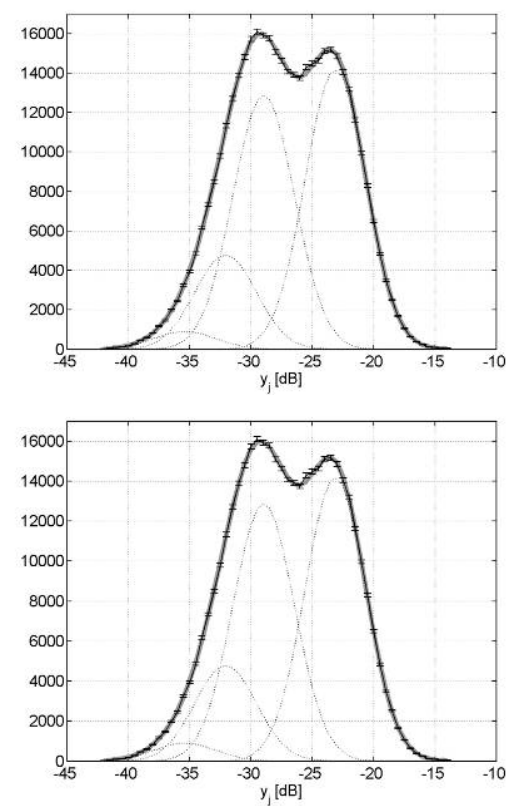

Fig.6 Histograms of measured backscatter data $\left(60^{\circ}\right.$ beam $)$ shown as the thick black lines. Also the fit for $m=5$ is shown (thick grey line), together with the five individual Gaussians (black dotted lines).

Based on these results, the boundaries between the different seafloor types are established (step 2 in section 2.2). These boundaries are indicated by the black squares in Fig. 7. The grey areas indicate the probabilities of incorrect decision, i.e., $\beta_{21}$ is the probability that $H_{1}$ is accepted, whereas actually $H_{2}$ holds true. $\beta_{12}$ is the probability that $H_{2}$ is accepted, whereas actually $H_{1}$ holds true. The decision matrix of the multiple-hypothesis-testing problem contains all probabilities of correct and incorrect decision and is given in Table 2.

Figure 8 shows the result of mapping the five seafloor types employing the backscatter data measured at both sides of the MBES system, where seafloor type 1 corresponds to the lowest and seafloor types 5 to the highest backscatter strength values. To highlight the presence of seafloor type 5 , positions corresponding to this seafloor type have been indicated with a slightly larger marker. The figure reveals clearly separated areas: the trench is filled with material corresponding to seafloor type 4 and surrounded by sediments with lower backscatter strength. Seafloor type 5 occurs in well-defined areas only.

\begin{tabular}{|c|l|l|l|l|}
\hline \multirow{2}{*}{$\begin{array}{c}\text { Seafloor } \\
\text { type }\end{array}$} & \multicolumn{3}{|c|}{ Side 1 } & \multicolumn{2}{c|}{ Side 2 } \\
\cline { 2 - 5 } & $\bar{y}_{k}$ & $\sigma_{y_{k}}$ & $\bar{y}_{k}$ & $\sigma_{y_{k}}$ \\
\hline 1 & -36.4 & 1.9 & -35.4 & 2.5 \\
\hline 2 & -32.5 & 2.1 & -32.1 & 2.5 \\
\hline 3 & -28.6 & 2.5 & -29.0 & 2.5 \\
\hline 4 & -22.5 & 2.4 & -23.1 & 2.5 \\
\hline 5 & -14.0 & 1.7 & -12.6 & 1.7 \\
\hline
\end{tabular}

Table 1 Means and standard deviation per seafloor type obtained from the fitting procedure (both in $\mathrm{dB}$ ).

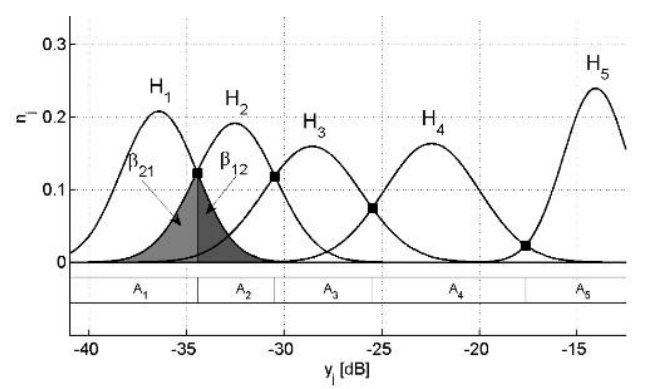

Fig.7 The Gaussian PDF for each seafloor type $H_{k}$. Black squares indicate the intersections of these functions. $A_{k}$ are the resulting acceptance regions for each $H_{k}$. Also indicated are examples of probabilities of wrong decision $\left(\beta_{12}, \beta_{21}\right)$.

\begin{tabular}{|l|c|c|c|c|c|}
\hline & $\begin{array}{c}H_{1} \\
\text { true }\end{array}$ & $\begin{array}{c}H_{2} \\
\text { true }\end{array}$ & $\begin{array}{c}H_{3} \\
\text { true }\end{array}$ & $\begin{array}{c}H_{4} \\
\text { true }\end{array}$ & $\begin{array}{c}H_{5} \\
\text { true }\end{array}$ \\
\hline Accept $H_{1}$ & $\mathbf{0 . 8 4}$ & 0.18 & 0.01 & 0 & 0 \\
\hline Accept $H_{2}$ & 0.15 & $\mathbf{0 . 6 6}$ & 0.21 & 0 & 0 \\
\hline Accept $H_{3}$ & 0 & 0.16 & $\mathbf{0 . 6 7}$ & 0.11 & 0 \\
\hline Accept $H_{4}$ & 0 & 0 & 0.11 & $\mathbf{0 . 8 7}$ & 0.02 \\
\hline Accept $H_{5}$ & 0 & 0 & 0 & 0.02 & $\mathbf{0 . 9 8}$ \\
\hline
\end{tabular}

Table 2 Decision matrix: Probabilities of correct decision are shown in bold on the diagonal.

It should be noted here that step 3 described in section 2.2 has been omitted, Therefore, Fig. 8 does not specify seafloor type, but acoustic class. Further analysis is needed to determine the seafloor types, corresponding to these different classes. As a first step the acoustic classification is compared with the video recordings. 


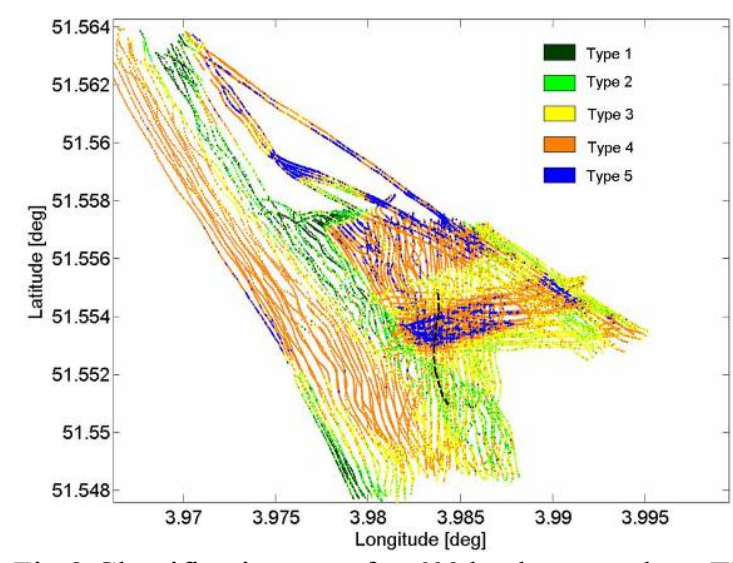

Fig. 8 Classification map for $60^{\circ}$ backscatter data. The black dashed line denotes the video track selected for comparison with classification data

\subsection{Comparison with the video recordings}

One particular video track that crossed the mussel culture spots from South to North was selected for further analysis (see Fig. 8). Figure 9 A presents the water depths along this track as a function of the latitude and across-track distance $X$. It can be seen that the track starts with a series of rippled structures. Figure $9 \mathrm{~B}$ shows the corresponding measured backscatter strengths. The white line, at $X$ is $\sim-10 \mathrm{~m}$, indicates the positions at which the 60 degree backscatter measurements have been taken (side 1). Figure $9 \mathrm{C}$ presents these measurements, again plotted as a function of latitude. The background of this figure shows the backscatter acceptance regions $A_{k}$ of the five different seafloor types in colors equal to those used for the map of Fig. 8. This plot clearly shows the effect of the rippled structures at the beginning of the track, where the backscatter strength shows large variations. The middle of the track shows the beginning of a new seafloor type with higher values for the backscatter strength. Along this part of the track a significant number of backscatter measurements are classified as type 5, i.e., the type corresponding to the highest values for the backscatter strength. Towards the end of the track, backscatter values are decreasing again. However, they remain higher than at the beginning of the track. Figure $9 \mathrm{D}$ shows the results of the video analysis as the percentage mussel coverage as a function of latitude. Comparing it with Fig. $9 \mathrm{C}$ indicates that the high backscatter values of Fig. $9 \mathrm{C}$ correspond to a high mussel coverage. This indicates that the well-separated seafloor type 4 and type 5 areas of Fig. 9 are actually mussel beds.

\section{Conclusion}

For the area considered the classification method indicates five different seafloor types to be present. Part of the area contains cultured mussel spots. From a comparison of the acoustic classification results with an analysis of video recordings it is concluded that the MBES classification method described in this paper is capable of discriminating areas with high mussel coverage. The mussel areas are clearly separated from the other areas.

\section{Acknowledgments}

The authors would like to thank Bernd Peters for driving the GKSS research vessel "Storch" and his technical assistance in setting up the MBES and positioning system. We also would like to thank the staff of the Netherlands Institute of Ecology, Centre for Estuarine and Marine Ecology in Yerseke, The Netherlands, for their kind hosting and immense technical support. The work was partly funded by the EU project MaBenE (grant EVK3-CT-200200071). This research is supported by the Dutch Technology Foundation STW, applied science division of NWO and the Technology Program of the Ministry of Economic Affairs.

A

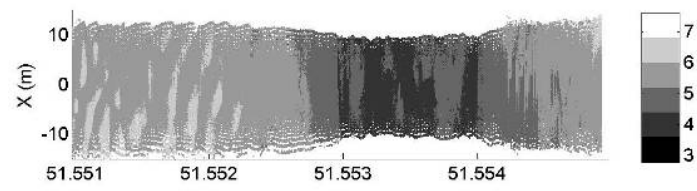

$\mathrm{B}$

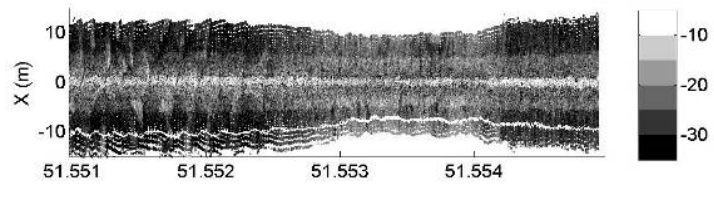

C
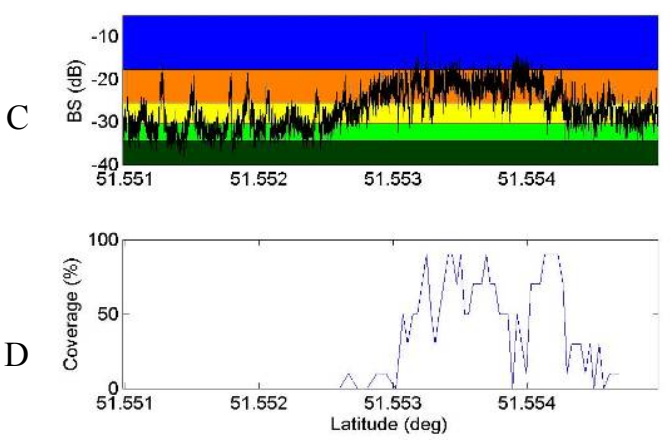

Fig.9 A) Water depth versus latitude and across-track distance $X$. B) Corresponding backscatter measurements. The white line at $X$ is $\sim-10 \mathrm{~m}$ indicates the positions at which the $60^{\circ}$ backscatter measurements were taken. C) Measured backscatter strengths. The background shows the five backscatter acceptance regions $A_{k}$. D) Mussel coverage determined from the video recordings analysis.

\section{References}

[1] Dick G. Simons and Mirjam Snellen, "A Bayesian approach to seafloor classification using multi-beam backscatter data", submitted to the Journal of Applied Acoustics

[2] Riethmüller, R., L. A. van Duren, C. Häse, T. Ysebaert, P. J. Wiles and P. M. J. Herman, "Vertical distribution of suspended matter in relation to turbulence and mussel filtration in a tidally dominated estuary", Report to the European Union, MaBenE Deliverable D2.2b, 2006

[3] C. Häse, M. Heineke, B. Peters, R. Riethmüller, R. Vorberg, "Fächerecholottechnik zur Kartierung und Sedimenterkennung in der Oosterschelde -Vermessung im Rahmen des EU-Projektes MaBenE", internal report by GKSS-Forschungszentrum Geesthacht $\mathrm{GmbH}$, Institut für Küstenforschung 